\title{
Decay solution for the renewal equation with diffusion
}

\author{
Boumediene Abdellaoui and Tarik Mohamed Touaoula
}

\begin{abstract}
In this paper we consider age structured equation with diffusion under nonlocal boundary condition and nonnegative initial data. We prove existence, uniqueness and the positivity of the solution to the above problem. Our main result is to get an exponential decay of the solution for large times toward such a study state. To this end we prove a weighted Poincaré-Wirtinger's type inequality in unbounded domain.
\end{abstract}

Mathematics Subject Classification (2000). 35B40, 35F10, 92D25.

Keywords. Cell division equations, Growth processes, General relative entropy, Asymptotic analysis.

\section{Introduction}

This paper deals with the following equation

$$
\left\{\begin{array}{l}
n_{t}(t, x)+n_{x}(t, x)+d(x) n(t, x)=n_{x x}, \quad t \geq 0, \quad x \geq 0 \\
n(t, 0)-n_{x}(t, 0)=\int_{0}^{\infty} B(y) n(t, y) d y, \text { and } n(0, x)=n^{0}(x) \in L^{1}\left(\mathbb{R}^{+}\right),
\end{array}\right.
$$

where $d$ and $B$ are nonnegative functions under a suitable hypotheses to be given later. Problem (1.1) arises in many applications, see for instance $[3,4,7$, $8,14]$ and the references therein. In particular problem (1.1) is considered as a basic model for age-structured populations. Denoting by $n(t, x)$, the cell density of "biological" age $x$ at time $t$, then the cells grow at a constant rate and divide into daughter cells at a rate $B(x)$. The variable $x$ can be considered as a biological age and can vary according to certain proteins causing degradation or recovery caused by external factors. This motivates the diffusion term in the model. In $[1,2]$, the authors propose to define the 'biological age' according to the DNA content and diffusion accounts for its variability. 
An interesting feature of this problem is to get the asymptotic behavior of $n(t, x)$, that gives the invasive capacity of the population and thus a fitness measure of population under different rates. For this, and as we will see later, the long time behavior of $n$ is directly linked with the existence of solution $(\lambda, N, \phi)$ to the associated eigenproblem. More precisely we will consider the following eigenproblem

$$
\left\{\begin{array}{l}
N^{\prime}(x)+(d(x)+\lambda) N(x)=N^{\prime \prime}(x), \quad x \geq 0 \\
N(0)-N^{\prime}(0)=\int_{0}^{\infty} B(y) N(y) d y \\
\int_{0}^{\infty} N(x) d x=1, \quad \text { and } N \geq 0
\end{array}\right.
$$

and its adjoint version

$$
\left\{\begin{array}{l}
-\phi^{\prime}(x)+(d(x)+\lambda) \phi(x)=\phi^{\prime \prime}(x)+B(x) \phi(0) \\
\phi^{\prime}(0)=0 \\
\int_{0}^{\infty} N(x) \phi(x) d x=1, \text { and } \phi \geq 0
\end{array}\right.
$$

with $\lambda$, the first eigenvalue called sometimes in Biology the Malthus parameter. The density $N$ plays the role of the so called stable steady dynamics, that is after a time renormalization, all solutions to (1.1) converge to a multiple $N$, the normalized solution of (1.2).

Notice that $\phi$ plays a fundamental role in the dynamics of (1.1), its allows to define a conservation law for the equation for $n(t, x)$ in the sense that

$$
\int_{0}^{\infty} n(t, x) e^{-\lambda t} \phi(x) d x=\int_{0}^{\infty} n^{0}(x) \phi(x) d x \quad \text { for all } t>0 .
$$

Throughout this paper, we assume that $B$ and $d$ are nonnegative continuous function such that

$$
0<B_{m} \leq B(x) \leq B_{M} \text { and } 0<d_{m} \leq d(x) \leq d_{M},
$$

and

$$
\max _{x \in \mathbb{R}^{+}}(B(x)-d(x))=\alpha, \text { and } \min _{x \in \mathbb{R}^{+}}(B(x)-d(x))=\beta,
$$

where $B_{m}, B_{M}, d_{m}, d_{M}, \alpha, \beta$ are positive constants.

The paper is organized as follows. In Sect. 2 we prove the existence, uniqueness and positivity of the solutions to problems (1.1), (1.2) and (1.3). In the first part of Sect. 2 we prove the existence of positive solution to (1.1) using approximating problem and a priori estimates. Section 2.1 is devoted to prove the existence and the uniqueness of positive solutions to (1.2) and (1.3).

In Sect. 3 we establish the main result concerning the asymptotic behavior of the solution to problem (1.1) as $t \rightarrow \infty$, moreover using a weighted Poincaré-Wirtinger type inequality we prove an exponential decay of $n(x, t)$, the solution of (1.1).

At the end of the paper we prove the weighted Poincaré-Wirtinger inequality used in Sect. 3. This result is important in itself, hence by proving fine properties of $N$ and $\phi$, solutions of (1.2) and (1.3) respectively, we get Poincaré-Wirtinger inequality in the unbounded set $[0, \infty)$. 


\section{Existence results}

The purpose of this section is to prove the existence, uniqueness and positivity of the solution to problem (1.1). More precisely we have the following theorem

Theorem 2.1. Assume that the assumptions (1.5) and (1.6) hold, then for all positive initial datum $n_{0} \in L^{1}\left(\mathbb{R}^{+}\right) \cap L^{2}\left(\mathbb{R}^{+}\right)$, there is a unique solution $n$ to problem (1.1) belongs to $\mathcal{C}\left([0, \infty) ; L^{2}\left(\mathbb{R}^{+}\right)\right) \cap L^{1}\left((0, T) \times \mathbb{R}^{+}\right) \cap$ $L^{2}\left(0, T ; W^{1,2}\left(\mathbb{R}^{+}\right)\right)$for all $T>0$.

To prove the existence result we will argue by approximation, namely we consider the case of bounded domain $[0, R]$ and then we pass to the limit in $R$. Let begin by proving the next Lemma.

Lemma 2.2. The following problem

$$
\left\{\begin{array}{l}
n_{t}(t, x)+n_{x}(t, x)+(d(x)+\mu) n(t, x)=n_{x x}, \quad t \geq 0, \quad x \in(0, R), \\
n(t, 0)-n_{x}(t, 0)=\int_{0}^{R} B(y) n(t, y) d y, \text { and } n(t, R)=0, \\
n(0, x)=n^{0}(x) .
\end{array}\right.
$$

has a unique positive solution $n_{R}$, moreover if $R_{1} \leq R_{2}$, then $n_{R_{1}} \leq n_{R_{2}}$.

Proof. To prove the existence of a positive solution to (2.2) we use the Piccard Banach fixed point theorem in the Banach space $X_{R}=\mathcal{C}\left([0, T], L^{1}(0, R)\right)$ with $\|\phi\|_{X_{R}}=\sup _{t \in[0, T]} \int_{0}^{R}|\phi(x, t)| d x$ and $\mu>0$ to be chosen later. More precisely given $m \in X_{R}$, we define $n:=S(m)$ as the solution to problem

$$
\left\{\begin{array}{l}
n_{t}(t, x)+n_{x}(t, x)+(d(x)+\mu) n(t, x)=n_{x x}, \text { in }(0, T) \times(0, R), \\
n(t, 0)-n_{x}(t, 0)=\int_{0}^{R} B(y) m(t, y) d y, n(t, R)=0, \\
n(0, x)=n^{0}(x), x \in(0, R) .
\end{array}\right.
$$

It is clear that $\left.n \in L^{2}\left([0, T], W^{1,2}(0, R)\right)\right) \cap X_{R}$. For $m_{1}, m_{2} \in X_{R}$ we consider $n_{1}:=S\left(m_{1}\right), n_{1}:=S\left(m_{2}\right)$. Let $n=n_{1}-n_{2}, m=m_{1}-m_{2}$, it follows that

$$
\left\{\begin{array}{l}
n_{t}(t, x)+n_{x}(t, x)+(d(x)+\mu) n(t, x)=n_{x x}, \text { in }(0, T) \times(0, R), \\
n(t, 0)-n_{x}(t, 0)=\int_{0}^{R} B(y) m(t, y) d y, n(t, R)=0, \\
n(0, x)=0 .
\end{array}\right.
$$

Multiplying Eq. (2.3) by $\operatorname{sgn}(n)$ and integrating in $x$,

$$
\frac{d}{d t} \int_{0}^{R}|n| d x+\int_{0}^{R}(d(x)+\mu)|n(t, x)| d x \leq \int_{0}^{R} B(y)|m(t, y)| d y,
$$

after integration over $\left(0, T_{1}\right), T_{1} \leq T$,

$$
\int_{0}^{R}\left|n\left(T_{1}, x\right)\right| d x+\int_{0}^{T_{1}} \int_{0}^{R}(d(x)+\mu)|n(t, x)| d x \leq B_{M} T|| m \|_{X_{R}}
$$

and then $\|n\|_{X_{R}} \leq B_{M} T\|m\|_{X_{R}}$. Hence choosing $T$ such $T B_{M}<1$ we obtain that the operator $S$ is strict contraction in Banach space $X_{R}$ which proves the existence of a unique fixed point $n_{R}$.

As usual we can iterate the operator on $[T, 2 T],[2 T, 3 T], \ldots$ since the condition on $T$ does not depend on the iteration. With this iteration process, we have built a solution in $\mathcal{C}\left([0, T], L^{1}(0, R)\right)$. Concerning the positivity of the solution, 
we multiply (2.2) by the negative part of $n_{R}$ and after integration, we obtain that the solution $n_{R} \geq 0$. The strong Maximum principle allows us to get the strict positivity of $n_{R}$. Let $R_{1} \leq R_{2}$, and consider the corresponding solutions $n_{R_{1}}$ and $n_{R_{2}}$. It is clear that $n_{R_{2}}$ is a supersolution to the $n_{R_{1}}$-problem. Hence using the comparison principle we obtain that $n_{R_{1}} \leq n_{R_{2}}$. Therefore the result follows.

We return now to prove the main existence Theorem.

Proof of Theorem 2.1. Let $n_{R}$ the solution to problem (2.2) obtained above. We define $n_{R}$ for $(t, x) \in(0, T) \times(R, \infty)$ by setting set $n_{R}(t, x)=0$. Then $n_{R} \in X \equiv L^{1}\left((0, T) \times \mathbb{R}^{+}\right) \cap L^{2}\left(0, T ; W^{1,2}\left(\mathbb{R}^{+}\right)\right)$. We know that

$$
\frac{d}{d t} \int_{0}^{R} n_{R}(t, x) d x+\int_{0}^{R}(d(x)+\mu) n_{R}(t, x) d x \leq B_{M} \int_{0}^{R} n_{R}(t, y) d y .
$$

Choosing $\mu>B_{M}$, it follows that

$$
\frac{d}{d t} \int_{0}^{R} n_{R}(t, x) d x+\int_{0}^{R}\left(d(x)+\mu-B_{M}\right) n_{R}(t, x) d x \leq 0 .
$$

Hence from Gronwall lemma we get

$$
\int_{0}^{R} n_{R}(t, x) d x \leq C e^{-c_{1} t}, \quad c_{1}>0 .
$$

Thus we conclude that $n_{R}$ is uniformly bounded in $L^{1}\left((0, T) \times \mathbb{R}^{+}\right)$.

Therefore using the monotonicity of the sequence $\left\{n_{R}\right\}$ we get the existence of $n \in L^{1}\left((0, T) \times \mathbb{R}^{+}\right)$such that $n_{R} \uparrow n$ as $R \rightarrow \infty$.

Taking $n_{R}$ as a test function in (2.1), after integration there result that

$\frac{1}{2} \frac{d}{d t} \int_{0}^{R} n_{R}^{2} d x+\int_{0}^{R}(d(x)+\mu)\left|n_{R}(t, x)\right|^{2} d x+\int_{0}^{R}\left|\left(n_{R}\right)_{x}(t, x)\right|^{2} d x \leq C \frac{B_{M}^{2}}{2} e^{-2 c_{1} t}$,

by integration in the time we get

$$
\begin{aligned}
& \int_{0}^{R}\left|n_{R}(t, x)\right|^{2} d x+\int_{0}^{t} \int_{0}^{R}(d(x)+\mu)\left|n_{R}(t, x)\right|^{2} d x d t+\int_{0}^{t} \int_{0}^{R}\left|\left(n_{R}\right)_{x}(t, x)\right|^{2} d x \\
& \quad \leq \frac{1}{2}\left\|n_{0}\right\|_{L^{2}}^{2}+C
\end{aligned}
$$

where $C$ is a positive constant. Thus $\left\|n_{R}\right\|_{L^{2}\left(0, T ; W^{1,2}\left(\mathbb{R}^{+}\right)\right)} \leq C$ and then $n_{R} \rightarrow$ $n$ weakly in $L^{2}\left(0, T ; W^{1,2}\left(\mathbb{R}^{+}\right)\right)$.

Therefore classical regularity result of parabolic equation allows us to pass to the limit in the boundary condition to conclude that $n$ solves problem (1.1). To get the uniqueness result for problem (1.1), we suppose that $n_{1}, n_{2} \in X$ are two solution of (1.1), then $w=n_{1}-n_{2}$, solves (1.1) with $w(0, x)=0$. Hence multiply the equation of $w$ by $\operatorname{sgn}(w)$, after integrating over $(0, \infty)$, we obtain

$$
\int_{0}^{\infty}|w|_{t}(t, x) d x+\int_{0}^{\infty}\left(d_{m}+\mu\right)|w(t, x)| d x \leq B_{M} \int_{0}^{\infty}|w(t, x)| d x
$$


thus

$$
\int_{0}^{\infty}|w|_{t}(t, x) d x+\alpha \int_{0}^{\infty}|w(t, x)| d x \leq 0
$$

and then by the Gronwall lemma we conclude that $w=0$. Hence the result follows.

\subsection{Mathematical analysis of the eigenvalue problem}

In this subsection we prove the existence of the first eigenvalue and the corresponding positive eigenvector for the problem (1.2) and (1.3). More precisely we have the following theorem.

Theorem 2.3. Assume that $B, d \in \mathcal{C}\left(\mathbb{R}^{+}\right)$. There is a unique solution $(\lambda, N, \phi)$ to problems (1.2) and (1.3) with $N \in W^{2,2}\left(\mathbb{R}^{+}\right)$and $\phi \in W_{\text {loc }}^{2,2}\left(\mathbb{R}^{+}\right) \cap L^{\infty}\left(\mathbb{R}^{+}\right)$. Moreover $\beta \leq \lambda \leq \alpha$.

Proof. As above, in order to prove Theorem 2.3 we first consider the case of a bounded domain $x \in[0, R]$, and then pass to the limit. Let $\lambda_{R}$ be first eigenvalue to problem

$$
\left\{\begin{array}{l}
N_{R}^{\prime}(x)+\left(d(x)+\lambda_{R}\right) N_{R}(x)=N_{R}^{\prime \prime}(x), \quad x \in(0, R), \\
N_{R}(0)-N_{R}^{\prime}(0)=\int_{0}^{R} B(y) N_{R}(y) d y, \\
N_{R}(R)=0 \text { and } \int_{0}^{R} N_{R}(x) d x=1,
\end{array}\right.
$$

We note $N_{R}$ the corresponding eigenfunction. Consider $\phi_{R}$, the solution to the corresponding dual problem, namely,

$$
\left\{\begin{array}{l}
-\phi_{R}^{\prime}(x)+\left(d(x)+\lambda_{R}\right) \phi_{R}(x)=\phi_{R}^{\prime \prime}(x)+B(x) \phi_{R}(0), \quad x \in(0, R), \\
\phi_{R}^{\prime}(0)=0, \\
\phi_{R}(R)=0, \text { and } \int_{0}^{R} N_{R}(x) \phi_{R}(x) d x=1 .
\end{array}\right.
$$

Notice that the existence of $\left(\lambda_{R}, N_{R}, \phi_{R}\right)$ could be obtained using the contraction mapping principle and the weak-Krein-Rutmann Theorem see for instance [9] (Appendix to Chapter VIII, Volume 5 p 223). We prove now the main a priori estimates. We will divide the proof in two main steps.

The first step We begin by proving a priori estimate on $N_{R}$.

Integrating the equation of $N_{R}$ over $(0, R)$ we get

$$
\lambda_{R}=\int_{0}^{R}(B(x)-d(x)) N_{R}(x) d x+N_{R}^{\prime}(R)
$$


Using the fact that $N_{R}^{\prime}(R) \leq 0$ and the normalization condition on $N_{R}$, it follows that

$$
\lambda_{R} \leq \alpha
$$

From (2.7), there result that

$$
\lambda_{R} \geq \beta+N_{R}^{\prime}(R) .
$$

Now using $x$ as a test function in the equation of $N_{R}$, and integrate over $(0, R)$

$$
-R N_{R}^{\prime}(R)+\int_{0}^{R}\left(d(x)+\lambda_{R}\right) x N_{R}(x) d x=1+N_{R}(0),
$$

hence

$$
-R N_{R}^{\prime}(R) \leq 1+N_{R}(0) .
$$

Notice that, the equation of $N_{R}$ can be written as

$$
-\left(e^{-x} N_{R}^{\prime}\right)^{\prime}+\left(d(x)+\lambda_{R}\right) N_{R} e^{-x}=0,
$$

then $e^{-x} N_{R}^{\prime}$ is a increasing function, which leads to $N_{R}^{\prime}(0) \leq 0$. Moreover by the boundary condition we get $N_{R}(0) \leq B_{M}$. Combining this inequality with (2.7) and (2.8) we find

$$
\lambda_{R} \geq \beta-\frac{1}{R}\left(1+B_{M}\right) .
$$

We establish now the uniform bounds of $N_{R}$. We claim that $N_{R}$ satisfies

$$
\left\|N_{R}\right\|^{2} \leq \frac{1}{2} \max \left(\frac{1}{\lambda_{R}}, 1\right) B_{M}^{2} .
$$

To prove the claim we use $N_{R}$ as a test function in (2.5), by integration over $(0, R)$, we get

$$
\begin{aligned}
\int_{0}^{R}\left|N_{R}^{\prime}\right|^{2} d x+\int_{0}^{R}\left(d(x)+\lambda_{R}\right)\left|N_{R}\right|^{2} d x & \leq \frac{1}{2}\left(N_{R}(0)-N_{R}^{\prime}(0)\right)^{2} \\
& \leq \frac{1}{2}\left|\int_{0}^{R} B(x) N_{R}(x) d x\right|^{2} \\
& \leq \frac{1}{2} B_{M}^{2}
\end{aligned}
$$

thus

$$
\left\|N_{R}\right\|^{2} \leq \frac{1}{2} \max \left(\frac{1}{\lambda_{R}}, 1\right) B_{M}^{2} .
$$

Hence the claim follows. By setting $N_{R}(x)=0$ for $x \geq R$, then using the above estimate, letting $R \rightarrow 0$, up to a subsequence, we get the existence of $N \in W^{1,2}\left(\mathbb{R}^{+}\right)$such that $N_{R} \rightarrow N$ weakly in $W^{1,2}\left(\mathbb{R}^{+}\right)$. Using the compact inclusion of $W^{1,2}\left(\mathbb{R}^{+}\right)$in $\mathcal{C}[0, a]$ for all $a>0$, it follows that $N_{R}(0) \rightarrow N(0)$. Now from (2.5) we get also that $N_{R}^{\prime \prime}$ is uniformly bounded in $L^{2}(0, R)$, independently of $R$, thus $N_{R}^{\prime}(0) \rightarrow N^{\prime}(0)$. Passing to the limit in the $N_{R}$ (in 
the sense of distribution), we get easily that $N \in W^{2,2}\left(\mathbb{R}^{+}\right)$. We prove now $\int_{0}^{\infty} N_{R} d x \rightarrow \int_{0}^{\infty} N d x$. To do that, we set $w:=w_{R}=N_{R}-N$, then $w$ satisfies

$$
-w^{\prime \prime}+w^{\prime}+(d(x)+\lambda) w=\left(\lambda-\lambda_{R}\right) N_{R},
$$

after integration over $(0, R)$ we obtain

$$
\int_{0}^{R}(d(x)+\lambda) w d x=\left(\lambda-\lambda_{R}\right)+N_{R}^{\prime}(R)-N^{\prime}(R)+N(R)+w(0)-w^{\prime}(0),
$$

since $N_{R} \geq 0$ and $N_{R}(R)=0$, we have $N_{R}^{\prime}(R) \leq 0$. Then

$$
\int_{0}^{R}(d(x)+\lambda) w d x \leq\left(\lambda-\lambda_{R}\right)+N(R)-N^{\prime}(R)+w(0)-w^{\prime}(0) .
$$

Then, using the definition of $w$ and by the Fatou lemma it follows that

$$
\begin{aligned}
\lim _{R \rightarrow \infty} \int_{0}^{R}(d(x)+\lambda) N_{R} & \leq \int_{0}^{\infty}(d(x)+\lambda) N(x) d x \\
& \leq \liminf _{R \rightarrow \infty} \int_{0}^{R}(d(x)+\lambda) N_{R} d x
\end{aligned}
$$

Moreover since $N_{R} \rightarrow N$ weakly in $W^{1,2}\left(\mathbb{R}^{+}\right)$, then, up to subsequence $(d(x)+$ $\lambda) N_{R} \rightarrow(d(x)+\lambda) N$ a.e. and strongly in $L_{\text {loc }}^{2}\left(\mathbb{R}^{+}\right)$. Therefore, using the fact that $N \in W^{2,2}\left(\mathbb{R}^{+}\right)$, the estimate (2.11) and the Brezis-Lieb theorem, we conclude that $(d(x)+\lambda) N_{R} \rightarrow(d(x)+\lambda) N$ strongly in $L^{1}\left(\mathbb{R}^{+}\right)$. Hence $N_{R} \rightarrow N$ in $L^{1}\left(\mathbb{R}^{+}\right)$and

$$
\int_{0}^{\infty} N(x) d x=1
$$

The second step A priori estimates on $\phi_{R}$.

In order to prove a priori bounds of $\phi_{R}$, we consider the following auxiliary problem

$$
\left\{\begin{array}{l}
-\left(e^{x} \psi^{\prime}(x)\right)^{\prime}=N_{R}(x), \quad x \in(0, R) \\
\psi(R)=0 \text { and } \psi^{\prime}(0)=1
\end{array}\right.
$$

with $N_{R}$ is solution to $(2.5)$. It is clear that

$$
\psi(x)=\int_{x}^{R} N_{R}(\sigma) e^{-\sigma} d \sigma+e^{-x} \int_{0}^{x} N_{R}(\sigma) d \sigma-e^{-x},
$$

and $\psi(x) \leq N_{R}(x) e^{-x}$. We claim that the next estimates hold

$$
\begin{gathered}
\frac{1}{B_{M}+1} \leq \phi_{R}(0) \leq 1+d_{M}+\lambda_{R}, \\
\left\|\phi_{R}\right\|_{W_{l o c}^{1,2}\left(\mathbb{R}^{+}\right)} \leq M .
\end{gathered}
$$


and

$$
\left\|\phi_{R}\right\|_{L^{\infty}\left(\mathbb{R}^{+}\right)} \leq C
$$

where $M, C$ are a positive constants.

In order to prove the claim we write Eq. (2.6) in the form

$$
-\left(\phi_{R}^{\prime}(x) e^{x}\right)^{\prime}+\left(d(x)+\lambda_{R}\right) \phi_{R}(x) e^{x}=B(x) \phi_{R}(0) e^{x} .
$$

Using $\psi$ as a test function in (2.16), integrating over $(0, R)$, we get

$$
\begin{aligned}
& \int_{0}^{R} \phi_{R}^{\prime}(x) e^{x} \psi^{\prime}(x) d x+\int_{0}^{R}\left(d(x)+\lambda_{R}\right) \phi_{R}(x) e^{x} \psi(x) d x \\
& \quad=\phi_{R}(0) \int_{0}^{R} B(x) e^{x} \psi(x) d x,
\end{aligned}
$$

hence

$$
\begin{aligned}
& -\phi_{R}(0) \psi^{\prime}(0)+\int_{0}^{R} \phi_{R}(x)\left(-e^{x} \psi^{\prime}(x)\right)^{\prime} d x+\int_{0}^{R}\left(d(x)+\lambda_{R}\right) \phi_{R}(x) e^{x} \psi(x) d x \\
& =\phi_{R}(0) \int_{0}^{R} B(x) e^{x} \psi(x) d x
\end{aligned}
$$

then

$$
1+\int_{0}^{R}\left(d(x)+\lambda_{R}\right) \phi_{R}(x) e^{x} \psi(x) d x=\phi_{R}(0)\left(\int_{0}^{R} B(x) e^{x} \psi(x) d x+1\right),
$$

thus we conclude that

$$
1 \leq \phi_{R}(0)\left(\int_{0}^{R} B(x) e^{x} \psi(x) d x+1\right) \leq \phi_{R}(0)\left(B_{M} \int_{0}^{R} N_{R}(x) d x+1\right),
$$

which leads to

$$
\phi_{R}(0) \geq \frac{1}{B_{M}+1} .
$$

Concerning the upper bound of $\phi_{R}(0)$, by (2.17), using the fact that $\psi(x) e^{x} \leq$ $N(x)$, we get

$$
\begin{aligned}
\phi_{R}(0) & \leq 1+\left(d_{M}+\lambda_{R}\right) \int_{0}^{R} \phi_{R}(x) e^{x} \psi(x) d x \\
& \leq 1+\left(d_{M}+\lambda_{R}\right) \int_{0}^{R} \phi_{R}(x) N_{R}(x) d x \\
& \leq 1+d_{M}+\lambda_{R} .
\end{aligned}
$$

Multiplying (2.6) by $N_{R} \phi_{R}$, integrating over $(0, R)$ and after integration by parts we obtain

$$
\begin{aligned}
& \int_{0}^{R}\left|\phi_{R}^{\prime}(x)\right|^{2} N_{R}(x) d x+\frac{1}{2} \int_{0}^{R}\left(d(x)+\lambda_{R}\right) N_{R}(x)\left|\phi_{R}(x)\right|^{2} d x \\
& \quad=\phi_{R}(0) \int_{0}^{R} B(x) N_{R}(x) \phi_{R}(x) d x-\frac{1}{2} \phi_{R}(0) \int_{0}^{R} B(x) N_{R}(x) d x
\end{aligned}
$$


thus

$$
\begin{aligned}
\int_{0}^{R}\left|\phi_{R}^{\prime}(x)\right|^{2} N_{R}(x) d x+\frac{1}{2} \int_{0}^{R}\left(d(x)+\lambda_{R}\right) N_{R}(x)\left|\phi_{R}(x)\right|^{2} d x & \leq \phi_{R}(0) B_{M} \\
& \leq\left(1+d_{M}+\lambda_{R}\right) B_{M} .
\end{aligned}
$$

Hence $\left\|\phi_{R}\right\|_{H_{l o c}^{1}(0, R)} \leq M$. In order to prove $(2.15)$, we set $v(x) \equiv C$ where $C$ is a positive constant satisfying $C \geq \frac{B_{M} C_{1}}{d_{m}+\lambda_{R}}$ with $C_{1} \geq \phi_{R}(0)$. It is clear that

$$
\left\{\begin{array}{l}
-\phi_{R}^{\prime \prime}(x)-\phi_{R}^{\prime}(x)+\left(d(x)+\lambda_{R}\right) \phi_{R}(x) \leq B_{M} C_{1}, \\
\phi_{R}^{\prime}(0)=0, \quad \phi_{R}(R)=0,
\end{array}\right.
$$

and

$$
\left\{\begin{array}{l}
-v^{\prime \prime}(x)-v^{\prime}(x)+(d(x)+\lambda) v(x) \geq\left(d_{m}+\lambda_{R}\right) C \geq B_{M} C_{1} \\
v^{\prime}(0)=0, \quad v(R)=C
\end{array}\right.
$$

then the comparison principle allows us to get (2.15) and then the claim follows.

Hence we conclude that $\phi_{R}$ converge to $\phi$ a solution of (1.3) satisfying the same bounds as in the claim. Since $N_{R} \phi_{R} \leq C N_{R}$ and from a variation of Lebesgue Theorem, we deduce that

$$
\int_{0}^{\infty} N(x) \phi(x) d x=1 .
$$

The third step: The uniqueness result To complete the proof of the theorem we show that $(\lambda, N, \phi)$ is the unique solution to the main problem. Let $\left(\lambda_{1}, N_{1}, \phi_{1}\right)$ be another solution, we have

$$
N_{1}^{\prime \prime} \phi-N_{1} \phi^{\prime \prime}-\left(N_{1} \phi\right)^{\prime}+\left(\lambda-\lambda_{1}\right) N_{1} \phi=\phi(0) B(x) N_{1}(x),
$$

after integration by parts, we find

$$
\left(\lambda-\lambda_{1}\right) \int_{0}^{\infty} N_{1} \phi d x=0,
$$

which implies that $\lambda=\lambda_{1}$. Next we prove that $N=N_{1}$. We set $N_{2}=N_{1}-N$, then $N_{2}$ satisfies the following problem

$$
-N_{2}^{\prime \prime}+N_{2}^{\prime}+(d+\lambda) N_{2}=0
$$

First of all is not difficult to see that $N(0)>0$. We claim that $\lambda$ is simple, indeed let $W(x)=N_{1}(x)-\frac{N_{1}(0)}{N(0)} N(x)$, then $W$ satisfies the same equation of $(2.20)$ and $W(0)=0$, then $W \equiv 0$, henceforth $\lambda$ is simple. Moreover, and without loss of generality we can assume that $N(0) \leq N_{1}(0)$, thus by the maximum principle we can deduce that $N_{2} \geq 0$ this imply that $N_{2} \equiv 0$ since $\int_{0}^{\infty} N_{2} d x=0$. Using the same computation as above we can prove that $\phi=\phi_{1}$. Hence the result follows. 


\section{The asymptotic behavior}

In this section we prove the asymptotic behavior of the solution, for more precise we prove the exponential decay for the renewal cell division equation. We first show that the Generalized Relative entropy see for instance [5, 6, 10, 12, 13] applies directly, Indeed a straightforward computation leads to the following result,

Theorem 3.1. Let $n$ be a solution to (1.1). The entropy equalities hold, namely, for all convex functions $H(\cdot)$, by setting $\tilde{n}=e^{-\lambda t} n$, we have

$$
\frac{\partial}{\partial t} \int_{0}^{\infty} N(x) \phi(x) H\left(\frac{\tilde{n}(t, x)}{N(x)}\right) d x=-D_{\text {diff }}^{H}(\tilde{n}(t))-D_{r e n}^{H}(\tilde{n}(t)) \leq 0,
$$

where the entropy dissipation due to diffusion and to the renewal terms are

$$
\begin{aligned}
D_{\text {diff }}^{H}(\tilde{n}(t))= & \int_{0}^{\infty} H^{\prime \prime}\left(\frac{\tilde{n}(t, x)}{N(x)}\right) N(x) \phi(x)\left(\frac{\partial}{\partial x} \frac{\tilde{n}(t, x)}{N(x)}\right)^{2} d x \geq 0, \\
D_{\text {ren }}^{H}(\tilde{n}(t))= & \phi(0) \int_{0}^{\infty}\left(H\left(\frac{\tilde{n}(t, x)}{N(x)}\right)-H\left(\frac{\tilde{n}(t, 0)}{N(0)}\right)\right. \\
& \left.-H^{\prime}\left(\frac{\tilde{n}(t, x)}{N(x)}\right)\left(\frac{\tilde{n}(t, x)}{N(x)}-\frac{\tilde{n}(t, 0)}{N(0)}\right)\right) B(x) N(x) d x \geq 0 .
\end{aligned}
$$

Remarking that the conservation law (1.4) can be obtained by choosing $H(u)=u$ in $(3.1)$.

Now we state the main result of this paper, namely an exponential rate of convergence to the renewal cell division equation.

Theorem 3.2. The solution to (1.1) converges to the steady state. Namely, setting $\tilde{n}=n e^{-\lambda t}$ and

$$
\rho=\int_{0}^{\infty} n_{0}(x) \phi(x) d x
$$

we have

$$
\int_{0}^{\infty}\left(\frac{\tilde{n}(t, x)-\rho N(x)}{N(x)}\right)^{2} N(x) \phi(x) d x \leq C_{0} e^{-C t} .
$$

with $C_{0}, C$ positive constants.

In order to prove Theorem 3.2 we need the following weighted PoincaréWirtinger inequality in $\mathbb{R}^{+}$that the proof is given in the Appendix.

Theorem 3.3. Let $u \in W^{1,2}\left(\mathbb{R}^{+} ; N \phi d x\right)$ be such that

$$
\int_{0}^{\infty} u(x) N(x) \phi(x) d x=0
$$

where $N, \phi$ are the solutions to (1.2) and (1.3) respectively. Then

$$
\int_{0}^{\infty}|u(x)|^{2} N(x) \phi(x) d x \leq C \int_{0}^{\infty}\left|u^{\prime}(x)\right|^{2} N(x) \phi(x) d x
$$

where $C$ a positive constant. 
Proof of Theorem 3.2. We set $k(t, x)=\tilde{n}(t, x)-\rho N(x)$, then $k(t, x)$ solves Eq. (1.1), thus the conservation law shows that

$$
\int_{0}^{\infty} k(t, x) \phi(x) d x=0 .
$$

For the case where convex function $H(u)=u^{2}$, the entropy equalities become

$$
\begin{aligned}
\frac{\partial}{\partial t} \int_{0}^{\infty} N(x) \phi(x)\left(\frac{k(t, x)}{N(x)}\right)^{2} d x= & -2 \int_{0}^{\infty} N(x) \phi(x)\left(\frac{\partial}{\partial x} \frac{k(t, x)}{N(x)}\right)^{2} d x \\
& -\phi(0) \int_{0}^{\infty}\left(\frac{k(t, x)}{N(x)}-\frac{k(t, 0)}{N(0)}\right)^{2} B(x) N(x) d x
\end{aligned}
$$

Thanks to Theorem 3.3, by setting $u(x)=\frac{k(t, x)}{N(x)}$, then from Gronwall lemma we deduce (3.2).

\section{Appendix: Poincaré-Wirtinger type inequality}

In this section we prove the weighted Poincaré-Wirtinger inequality used in Theorem 3.3. The main planing to get the desired result is the following:

(1) We elaborate some estimates on $N$ and $\phi$.

(2) We demonstrate that the constant associated to the weighted Poincaré inequality in bounded domain of the form $[0, R]$ is independent of $R$.

(3) We prove the weighted Poincaré-Wirtinger inequality in $\mathbb{R}^{+}$using a priori estimate and an auxiliary problem.

Let begin by proving the next lemma.

Lemma A.1. Let $N$ and $\phi$ be the solutions of the stationary and the adjoint problems respectively, namely

$$
\left\{\begin{array}{l}
N^{\prime}(x)+(d(x)+\lambda) N(x)=N^{\prime \prime}(x), \quad x \geq 0 \\
N(0)-N^{\prime}(0)=\int_{0}^{\infty} B(y) N(y) d y \\
\int_{0}^{\infty} N(x) d x=1, \quad \text { and } N \geq 0
\end{array}\right.
$$

and

$$
\left\{\begin{array}{l}
-\phi^{\prime}(x)+(d(x)+\lambda) \phi(x)=\phi^{\prime \prime}(x)+B(x) \phi(0) \\
\phi^{\prime}(0)=0 \\
\int_{0}^{\infty} N(x) \phi(x) d x=1, \text { and } \phi \geq 0 .
\end{array}\right.
$$

then

(1) $\left|\frac{N^{\prime}(x)}{N(x)}\right| \leq C$, moveover for $x$ large we have $N(x) \leq \beta e^{-\alpha x}$, with $C, \alpha, \beta$ positives.

(2) $\left|\frac{\phi^{\prime}(x)}{\phi(x)}\right| \leq C_{1}$, for some positive constant $C_{1}$.

Proof. Let $v(x)=\frac{N^{\prime}(x)}{N(x)}\left(\operatorname{resp} p(x)=\frac{\phi^{\prime}(x)}{\phi(x)}\right)$ then, using Eq. $((1.2))$ (resp. $((1.3)))$, we have 


$$
v^{\prime}=-v^{2}+v+d(x)+\lambda \quad\left(\operatorname{resp} \cdot p^{\prime}=-p^{2}-p+d(x)+\lambda-B \frac{\phi(0)}{\phi(x)}\right) .
$$

Since $d(x)+\lambda$ is bounded and using the fact that (A.1) is similar to a logistic equation, we get that $v$ (resp for $p$ ) has two possible alternatives:

1. Either $v$ and $v^{\prime}$ (resp. $p$ ) are bounded,

2. Or there exists $x_{0}<\infty$ s.t. $\lim _{x \rightarrow x_{0}} v(x)=-\infty\left(\right.$ resp. $\lim _{x \rightarrow x_{0}} p(x)=$ $-\infty)$.

Using the strong maximum principle we conclude that $N($ resp $\phi)$ is strictly positive in $\mathbb{R}^{+}$, then since $N \in W^{2,2}\left(\mathbb{R}^{+}\right)$, it follows that the second alternative can't be true. Thus $v$ (resp $p$ ) is bounded.

We prove now the exponential decay of $N$. Let $a_{1}, a_{2}$ be the roots of the algebraic equation $-s^{2}+s+\lambda+d_{m}=0$, where $d_{m}$ is given in (1.5), then

$$
a_{1}=\frac{1}{2}\left(1-\sqrt{1+4\left(d_{m}+\lambda\right)}\right), \quad a_{2}=\frac{1}{2}\left(1+\sqrt{1+4\left(d_{m}+\lambda\right)}\right) .
$$

We claim that $v(x) \leq a_{1}<0$ for all $x \in \mathbb{R}^{+}$. To prove the claim we argue by contradiction. Assume the existence of $x_{0}$ such that $a_{1}<v\left(x_{0}\right)$.

Notice that from (1.2), we have $e^{-x} N^{\prime}$ is increasing. Then, since $N \in$ $W^{2,2}\left(\mathbb{R}^{+}\right)$, we get $\lim _{x \rightarrow \infty} e^{-x} N^{\prime}(x)=0$. Thus $N^{\prime}(x) \leq 0$, and then $v(x) \leq 0$. Therefore $a_{1}<v\left(x_{0}\right) \leq 0$. From (A.1), there result that $v^{\prime}\left(x_{0}\right)>0$. Then by an iteration argument we conclude that $v^{\prime}>0$ in $\left(x_{0}, \infty\right)$ and then $v$ is increasing in $\left(x_{0}, \infty\right)$.

Let $l=\lim _{x \rightarrow \infty} v(x)$, It is clear that $a_{1}<l \leq 0$. Thus $-v^{2}(x)+v(x)+d(x)+\lambda \geq$ $c>0$ for $x>x_{1}$ with $x_{1}$ large enough.

Integrating (A.1), we get

$$
\int_{x_{1}}^{x} v^{\prime}(s) d s=\int_{x_{1}}^{x}\left(-v^{2}(s)+v(s)+d(s)+\lambda\right) d s \geq c\left(x-x_{1}\right) .
$$

Thus $v(x)-v\left(x_{1}\right) \geq c\left(x-x_{1}\right)$. Letting $x \rightarrow \infty$, we reach a contradiction with the fact that $a_{1} \leq l \leq 0$. Thus $v(x) \leq a_{1}<0$ and the claim follows. Now the exponential decay of $N$ is an easy consequence of the above estimate.

Let be state the following lemma which concern the Poincaré inequality, in a bounded domain, which plays a fundamental role to prove our main theorem.

Lemma A.2. There exists a constant $C>0$ such that for all $w \in W_{0}^{1,2}(0, R)$ we have

$$
\int_{0}^{R}|w(x)|^{2} N(x) \phi(x) d x \leq C \int_{0}^{R}\left|w^{\prime}(x)\right|^{2} N(x) \phi(x) d x,
$$

with $C$ independent of $R$.

To prove the above lemma we need to state the following theorem, for the proof see [11, page 13]. 
Theorem A.3. Setting

$$
F_{R}(x)=\int_{x}^{R} N(s) \phi(s) d s \int_{0}^{x} \frac{1}{N(s) \phi(s)} d s
$$

and $B(R)=\sup _{x \in(0, R)} F_{R}^{\frac{1}{2}}(x)$. Then the inequality

$$
\int_{0}^{R}|w(x)|^{2} N(x) \phi(x) d x \leq C \int_{0}^{R}\left|w^{\prime}(x)\right|^{2} N(x) \phi(x) d x
$$

holds for every $w \in W_{0}^{1,2}(0, R)$ if and only if

$$
B(R)<\infty .
$$

Moreover for the best possible constant $C$ in (A.3), the following estimate is satisfied

$$
B(R) \leq C \leq 2 B(R)
$$

Proof of Lemma A.2. Since $F_{R}$ is a continuous function, then to prove the lemma we have just to show the existence of $c_{1}, c_{2}>0$ such that $c_{1} \leq B(R) \leq$ $c_{2}$ for $R$ large. It is easy to see that $B(R) \geq c_{1}>0$ for $R>>1$. To get the desired result we have to show that $F_{R}(x) \leq C$ for all $x \in[0, R]$ and for all $R>1$. Let consider $F(x)=\int_{x}^{\infty} N(s) \phi(s) d s \int_{0}^{x} \frac{1}{N(s) \phi(s)} d s$, then we have

$$
F^{\prime}(x)=g(x)(k(x)-F(x)),
$$

with $g(x)=\frac{N(x) \phi(x)}{\int_{x}^{\infty} N(s) \phi(s) d s}>0$ and $k(x)=\left(\frac{\int_{x}^{\infty} N(s) \phi(s) d s}{N(x) \phi(x)}\right)^{2}$. Notice that from Lemma A.1 we get easily that $g$ is bounded.

Multiplying the equation of $N$ by $\phi$ and the equation of $\phi$ by $N$,

$$
\phi N^{\prime \prime}=\phi N^{\prime}+(d(x)+\lambda) \phi N
$$

and

$$
N \phi^{\prime \prime}=-N \phi^{\prime}+(d(x)+\lambda) N \phi-B(x) \phi(0) N,
$$

by subtracting the equations and integrating over $(x, \infty)$ we get

$$
\int_{x}^{\infty} \phi(s) N^{\prime \prime}(s) d s-\int_{x}^{\infty} N \phi^{\prime \prime}(s) d s=\int_{x}^{\infty}(N(s) \phi(s))^{\prime} d s+\phi(0) \int_{x}^{\infty} B(s) N(s) d s,
$$

since $\left|\frac{\phi^{\prime}}{\phi}\right| \leq C$, then $\lim _{x \rightarrow \infty}\left|\phi^{\prime}(x)\right| \leq C \lim _{x \rightarrow \infty} \phi(x) \leq M$, thus

$$
N(x) \phi(x)+N(x) \phi^{\prime}(x)-\phi(x) N^{\prime}(x)=\phi(0) \int_{x}^{\infty} B(s) N(s) d s .
$$

Using the fact that $N(x) \geq C N(x) \phi(x)$, it follows that

$$
N(x) \phi(x)+N(x) \phi^{\prime}(x)-\phi(x) N^{\prime}(x) \geq c \phi(0) \int_{x}^{\infty} N(s) \phi(s) d s,
$$

thus we have

$$
0 \leq k(x) \leq\left(\frac{1+\phi^{\prime} / \phi-N^{\prime} / N}{c \phi(0)}\right)^{2}
$$


Using Lemma A.1, we find that $0 \leq k(x) \leq K<\infty$ and then

$$
F(x) \leq K \text {. }
$$

Let now $u \in W^{1,2}\left(\mathbb{R}_{+}, N \phi d x\right)$ with

$$
\int_{0}^{\infty} N(x) \phi(x) u(x) d x=0
$$

and consider the following auxiliary problem

$$
\left\{\begin{array}{l}
-\left(N \phi w^{\prime}\right)^{\prime}(x)=N(x) \phi(x) u(x) \\
w(0)=0, \quad w \in W^{1,2}\left(\mathbb{R}^{+} ; N \phi d x\right)
\end{array}\right.
$$

Let begin by proving the next existence result.

Lemma A.4. There exists a unique solution $w$ to problem (A.6) such that

$$
\int_{0}^{\infty} w^{2}(x) N(x) \phi(x) d x \leq C \int_{0}^{\infty}\left|w^{\prime}\right|^{2}(x) N(x) \phi(x) d x,
$$

where $C$ is the same constant as in Lemma A.2.

Proof. Consider $w_{R}$, the unique solution to the following approximate problem

$$
\left\{\begin{array}{l}
-\left(N \phi w_{R}^{\prime}\right)^{\prime}(x)=N(x) \phi(x) u(x), \quad x \in(0, R) \\
w_{R}(0)=w_{R}(R)=0
\end{array}\right.
$$

It is clear that $w_{R}(x)=-\int_{0}^{R} G(x, y) N(y) \phi(y) u(y) d y$ where

$$
G(x, y)= \begin{cases}\left(1-\frac{\int_{0}^{y} \frac{1}{N(\sigma) \phi(\sigma)} d \sigma}{\int_{0}^{R} \frac{1}{N(\sigma) \phi(\sigma)} d \sigma}\right) \int_{0}^{x} \frac{1}{N(\sigma) \phi(\sigma)} d \sigma & \text { if } 0 \leq x \leq y, \\ \left(1-\frac{\int_{0}^{x} \frac{1}{N(\sigma) \phi(\sigma)} d \sigma}{\int_{0}^{R} \frac{1}{N(\sigma) \phi(\sigma)} d \sigma}\right) \int_{0}^{y} \frac{1}{N(\sigma) \phi(\sigma)} d \sigma & \text { if } 0 \leq y \leq x \leq R .\end{cases}
$$

We set $w_{R}(x)=0$ for $x \geq R$. Using $w_{R}$ as a test function in (A.8) and using Cauchy-Schwarz inequality we obtain that

$$
\begin{aligned}
\int_{0}^{\infty}\left|w_{R}^{\prime}(x)\right|^{2} N(x) \phi(x) d x \leq & \left(\int_{0}^{\infty}|u(x)|^{2} N(x) \phi(x) d x\right)^{1 / 2} \\
& \times\left(\int_{0}^{\infty}\left|w_{R}(x)\right|^{2} N(x) \phi(x) d x\right)^{1 / 2} .
\end{aligned}
$$

From Lemma A.2, we get the existence of a positive constant $\bar{c}$ independent of $R$ such that

$$
\int_{0}^{\infty}\left|w_{R}^{\prime}(x)\right|^{2} N(x) \phi(x) d x \leq \bar{c},
$$

hence it follows that $w_{R} \rightarrow w$ weakly in $W^{1,2}\left(\mathbb{R}^{+} ; N \phi d x\right)$. Since $N(x) \phi(x)>0$ for all $x \in[0, \infty)$, there result that $w_{R} \rightarrow w$ weakly in $W_{l o c}^{1,2}\left(\mathbb{R}^{+}\right)$and then $w_{R} \rightarrow w$ strongly in $L_{l o c}^{2}([0, \infty))$. It is not difficult to see that $w$ solves (A.6) in a distribution sense with

$$
\int_{0}^{\infty}|w(x)|^{2} N(x) \phi(x) d x+\int_{0}^{\infty}\left|w^{\prime}(x)\right|^{2} N(x) \phi(x) d x \leq C .
$$


Since $\int_{0}^{\infty}|u(x) N(x) \phi(x)|^{2} d x \leq C_{1}$, then using the fact that $N \phi \in L^{1}(0, \infty) \cap$ $L^{\infty}(0, \infty)$, we conclude that $w^{\prime}(x) N(x) \phi(x) \in W^{1,2}\left(\mathbb{R}^{+}\right)$and then $\lim _{R \rightarrow \infty}$ $w^{\prime}(R) N(R) \phi(R)=0$.

We claim that $w_{R}^{\prime} \rightarrow w^{\prime}$ strongly in $L^{2}\left(\mathbb{R}^{+}, N \phi d x\right)$. To prove the claim we set $v_{R}=w_{R}-w$, then $v_{R} \rightarrow 0$ strongly in $L_{l o c}^{2}([0, \infty))$ and $\left\|v_{R}\right\|_{w^{1,2}\left(\mathbb{R}^{+}, N \phi d x\right)} \leq C$ for all $R$. It is clear that $v_{R}$ solves the problem

$$
\left\{\begin{array}{l}
-\left(N \phi v_{R}^{\prime}\right)^{\prime}(x)=0 \\
v_{R}(0)=0, \text { and } v_{R}(R)=-w(R) .
\end{array}\right.
$$

A direct computation shows that

$$
v_{R}(x)=-w(R) \frac{\int_{0}^{x} \frac{1}{N(s) \phi(s)} d s}{\int_{0}^{R} \frac{1}{N(s) \phi(s)} d s} \quad \text { if } x \leq R \text { and } v_{R}(x)=-w(x) \text { for } x \geq R .
$$

Using $v_{R}$ as a test function in (A.9) and integrating over $(0, R)$

$w_{R}^{\prime}(R) N(R) \phi(R) w(R)-w(R) N(R) \phi(R) w^{\prime}(R)+\int_{0}^{R}\left|v_{R}^{\prime}(x)\right|^{2} N(x) \phi(x) d x=0$.

To get the claim it is sufficient to prove that $\lim _{R \rightarrow \infty} w_{R}^{\prime}(R) w(R) N(R) \phi(R)=$ 0 and $\lim _{R \rightarrow \infty} w^{\prime}(R) w(R) N(R) \phi(R)=0$. Indeed using $w$ as test function in (A.8) and integrating over $(0, R)$, we get

$$
\begin{aligned}
& w_{R}^{\prime}(R) w(R) N(R) \phi(R)-w_{R}^{\prime}(0) w(0) N(0) \phi(0)+\int_{0}^{R} w_{R}^{\prime}(x) w^{\prime}(x) N(x) \phi(x) d x \\
& \quad=\int_{0}^{R} u(x) w(x) N(x) \phi(x) d x,
\end{aligned}
$$

integrating by part, yields

$w_{R}^{\prime}(R) w(R) N(R) \phi(R)=\int_{0}^{R}\left(w(x)-w_{R}(x)\right) u(x) N(x) \phi(x) d x \rightarrow 0, \quad$ as $R \rightarrow \infty$.

Consider now the function

$$
\theta_{R}(s)= \begin{cases}1, & \text { if } s \leq R \\ (1+R)-s, & \text { if } R \leq s \leq R+1 \\ 0, & \text { if } s \geq R+1\end{cases}
$$

Using $\theta_{R} w$ as a test function in (A.6) and integrating,

$$
\begin{aligned}
& \int_{0}^{R+1} N(x) \phi(x)\left|w^{\prime}(x)\right|^{2} \theta_{R}(x) d x+\int_{R}^{R+1} N(x) \phi(x) w(x) w^{\prime}(x) \theta_{R}^{\prime}(x) d x \\
& \quad=\int_{0}^{R} u(x) N(x) \phi(x) w(x) \theta_{R}(x) d x,
\end{aligned}
$$

so, passing to the limit

$$
\int_{0}^{\infty} N(x) \phi(x)\left|w^{\prime}(x)\right|^{2} d x=\int_{0}^{\infty} u(x) w(x) N(x) \phi(x) d x .
$$


Using $w$ as a test function in (A.6) we get

$$
\begin{aligned}
& \int_{0}^{R}\left|w^{\prime}(x)\right|^{2} N(x) \phi(x) d x-w^{\prime}(R) w(R) N(R) \phi(R) \\
& \quad=\int_{0}^{R} u(x) w(x) N(x) \phi(x) d x .
\end{aligned}
$$

Passing to the limit as $R \rightarrow \infty$, from (A.10) and (A.11) we find $\lim _{R \rightarrow \infty} w^{\prime}(R)$ $w(R) N(R) \phi(R)=0$. Therefore we conclude that $\int_{0}^{R}\left|v_{R}^{\prime}(x)\right|^{2} N(x) \phi(x) d x \rightarrow 0$ as $R \rightarrow \infty$ and then the claim follows. Notice that using the previous computation we can show that $v_{R} N \phi \rightarrow 0$ strongly in $W^{1,2}\left(\mathbb{R}^{+}\right)$.

Since $w_{R} \rightarrow w$ strongly in $L_{l o c}^{2}([0, \infty))$, then using the result of the claim, the Fatou's lemma and the weighted Poincaré inequality in (A.2), we obtain that

$$
\int_{0}^{R} w^{2}(x) N(x) \phi(x) d x \leq C \int_{0}^{R}\left|w^{\prime}\right|^{2}(x) N(x) \phi(x) d x,
$$

To prove the uniqueness result we consider $w_{1}, w_{2} \in W^{1,2}\left(\mathbb{R}^{+}, N \phi d x\right)$, two solution of problem (A.6). From the above discussion we get easily that $N \phi w_{i}^{\prime} \in W^{1,2}\left(\mathbb{R}^{+}\right)$and then $N(R) \phi(R) w_{i}(R) \rightarrow 0$ as $R \rightarrow \infty, i=1,2$. Setting $w=w_{1}-w_{2}$, then $\left(N \phi w^{\prime}\right)^{\prime}=0, w(0)=0$ and $w \in W^{1,2}\left(\mathbb{R}^{+}\right)$. Using $w$ as a test function in the above equation, by integration in $(0, \infty)$ it follows that $\int_{0}^{\infty}\left|w^{\prime}\right|^{2} N \phi d x=0$, hence $w^{\prime}=0$ and then $w \equiv C$. Since $w(0)=0$, it follows that $C=0$ and the Lemma A.4 is proved.

Now we are able to prove the main Theorem of the Appendix.

Theorem A.5. Assume that a function $u \in W^{1,2}\left(\mathbb{R}^{+} ; N \phi d x\right)$ satisfies

$$
\int_{0}^{\infty} u(x) N(x) \phi(x) d x=0
$$

where $N, \phi$ are solutions to (1.2) and (1.3) respectively. Then we have the following weighted Poincaré-Wirtinger inequality

$$
\int_{0}^{\infty}|u(x)|^{2} N(x) \phi(x) d x \leq C \int_{0}^{\infty}\left|u^{\prime}(x)\right|^{2} N(x) \phi(x) d x
$$

with $C$ a positive constant.

Proof. First of all, using the fact that $N(R) \phi(R) w^{\prime}(R) \rightarrow 0$ as $R \rightarrow \infty$, then integrating (A.6) over $(0, \infty)$, and in view of $\left(\right.$ A.5), it follows that $w^{\prime}(0)=0$. Using $u$ as a test function in (A.6), by integration we get

$$
\int_{0}^{\infty} w^{\prime}(x) u^{\prime}(x) N(x) \phi(x) d x=\int_{0}^{\infty}|u(x)|^{2} N(x) \phi(x) d x .
$$


From Cauchy-Schwarz inequality we obtain

$$
\begin{aligned}
\int_{0}^{\infty}|u(x)|^{2} N(x) \phi(x) d x \leq & \left(\int_{0}^{\infty}\left|u^{\prime}(x)\right|^{2} N(x) \phi(x) d x\right)^{1 / 2} \\
& \times\left(\int_{0}^{\infty}\left|w^{\prime}(x)\right|^{2} N(x) \phi(x) d x\right)^{1 / 2} .
\end{aligned}
$$

Multiplying Eq. (A.6) by $w$ and integrating over $(0, \infty)$, there result that

$$
\begin{aligned}
\int_{0}^{\infty}\left|w^{\prime}(x)\right|^{2} N(x) \phi(x) d x \leq & \left(\int_{0}^{\infty}|u(x)|^{2} N(x) \phi(x) d x\right)^{1 / 2} \\
& \times\left(\int_{0}^{\infty}|w(x)|^{2} N(x) \phi(x) d x\right)^{1 / 2}
\end{aligned}
$$

using (A.7) we get

$$
\int_{0}^{\infty}\left|w^{\prime}(x)\right|^{2} N(x) \phi(x) d x \leq C \int_{0}^{\infty}|u(x)|^{2} N(x) \phi(x) d x,
$$

consequently by (A.13) it holds

$$
\begin{aligned}
\int_{0}^{\infty}|u(x)|^{2} N(x) \phi(x) d x \leq & C\left(\int_{0}^{\infty}|u(x)|^{2} N(x) \phi(x) d x\right)^{1 / 2} \\
& \times\left(\int_{0}^{\infty}\left|u^{\prime}(x)\right|^{2} N(x) \phi(x) d x\right)^{1 / 2}
\end{aligned}
$$

finally

$$
\int_{0}^{\infty}|u(x)|^{2} N(x) \phi(x) d x \leq C_{1} \int_{0}^{\infty}\left|u^{\prime}(x)\right|^{2} N(x) \phi(x) d x .
$$

and Theorem A.5 is proved.

\section{Acknowledgments}

The authors would like to thank the referees for many helpful comments and suggestions that improve a last version of this paper. The second author wish to thank Pr. Benoît Perthame for very useful discussions and comments.

\section{References}

[1] Basse, B., Baguley, B.C., Marshall, E.S., Joseph, W.R., van Brunt, B., Wake, G., Wall, D.J.N.: A mathematical model for analysis of the cell cycle in cell lines derived from human tumors. J. Math. Biol. 47(4), 295-312 (2003)

[2] Brewer, J.W.: The age-dependent eigenfunctions of certain Kolmogorov equations of engineering, economics, and biology. Appl. Math. Model. 13, 47-57 (1989)

[3] Day, W.A.: Extensions of property of solutions of heat equation to linear thermoelasticity and other theories. Q. Appl. Math. 40, 319-330 (1983) 
[4] Metz, J.A.J., Diekmann, O.: The dynamics of phisiologically structured populations. LN in Biomathematics, vol. 68. Springer, Berlin (1986)

[5] Michel, P., Mishler, S., Perthame, B.: General entropy equations for structured population models and scattering. C. R. Math. Acad. Sci. Paris 338(9), 697-702 (2004)

[6] Michel, P., Mishler, S., Perthame, B.: General relative entropy inequality: an illustration on growth models. J. Math. Pures. Appl. 84(9), 1235-1260 (2005)

[7] Kerefov, A.A.: Non-local boundary value problems for parabolic equation. Differ. Ukravn. 15, 74-78 (1979)

[8] Lee, Y.H., Sherbakov, L., Taber, J., Shi, J.: Bifurcation diagrams of population models with nonlinear diffusion. J. Comput. Appl. Math. 194, 357-367 (2006)

[9] Dautray, R., Lions, J.L.: Analyse Mathématique et calcul numérique pour les sciences et les techniques. Masson, Paris (1988)

[10] Mishler, S., Perthame, B., Ryzhik, L.: Stability in a nonlinear population maturation model. Math. Model. Methods Appl. Sci. 12, 1751-1772 (2002)

[11] Opic, B., Kufner, A.: Hardy-type Inequalities. Longman Scientific and Technical, New York (1990)

[12] Perthame, B., Ryzhik, L.: Exponential decay for the fragmentation or cell division equation. J. Differ. Equ. 210, 155-177 (2005)

[13] Perthame, B.: Transport Equations in Biology. Birkhauser, Berlin (2007)

[14] Vabishchevich, P.N.: Non-local parabolic problems and the inverse heat-conduction problem. Differ. Ukravn. 17, 1193-1199 (1981)

B. Abdellaoui and T. M. Touaoula

Département de Mathématiques,

Université Aboubekr Belkaïd,

Tlemcen 13000, Algeria

e-mail: boumediene.abdellaoui@uam.es

T. M. Touaoula

e-mail: t_touaoula@yahoo.fr

Received: 22 January 2009.

Accepted: 16 December 2009. 